\title{
Studies on leaf and nut blight of cashew (Anacardium occidentale) caused by Cryptosporiopsis sp. in Tanzania
}

\section{Menge $D^{1}$ and Shomari $S^{2}$}

\author{
${ }^{1}$ Maasai Mara University P. O. Box 861-20500 Narok, Kenya \\ ${ }^{2}$ Cashew Research Programme, Naliendele Agricultural Research Institute (NARI), P.O. Box 509, Mtwara, Tanzania
}

Menge D, Shomari S 2016 - Studies on leaf and nut blight of cashew (Anacardium occidentale) caused by Cryptosporiopsis sp. in Tanzania. Plant Pathology \& Quarantine Plant 6(2), 201-205, Doi $10.5943 / \mathrm{ppq} / 6 / 2 / 8$

\begin{abstract}
Leaf and nut blight (Cryptosporiopsis sp.) of cashew is a very common and destructive disease in Tanzania. Symptoms coincide with flushing of shoots and flowering from July to December. Cashew leaf and nut blight infects young tender shoots, pseudo-fruits and nuts. Initially, the disease appears in the form of small, scattered brown spots on the leaf lamina. Later, these spots increase in size and coalesce covering larger leaf area, with dark brown margin. The first symptoms of the disease are chlorotic spots on both sides of the youngest tender leaves. Leaf spots vary in size, shape and colour between different varieties of cashew. The spots are brown, with a dark brown border, spreading necrotic lesions leading to leaf blight; circular, irregular or angular $1-2 \mathrm{~cm}$ in diameter; irregular roughened or corky lesions with eruption and necrosis of epidermal tissue, sometimes localized along veins. The diseased leaves curl and can be totally defoliated.
\end{abstract}

Keywords - cashew - leaf and nut blight - symptoms

\section{Introduction}

Cashew (Anacardium occidentale L.) is one of the most popular tree nuts on the regional and world markets because of its competitive price, long shelf life, relatively low fat content and excellent flavour. However, despite strong market demand, total production of raw nuts has decreased in recent years. Leaf and nut blight caused by Cryptosporiopsis sp. is a major limiting factor affecting cashew nut production in Tanzania, causing 48.4\% crop loss annually (ACRR 2006). Direct infection of young nuts is a major effect. The young nuts blacken and undergo abscission. The disease is most active during wet weather, especially during off-season rains, where severe infections affect the young flushing material (Sijaona et al. 2006). The Global Plant Clinic received a cashew inquiry from the Caribbean in 2007, which on further investigation proved to be the same fungus and disease found in Tanzania. The taxonomy of the pathogen is being studied (GPC 2010). Disease symptoms include angular leaf lesions, dark tan with a dark reddish brown margin, which are often vein limited and containing conidiomata (Sijaona et al. 2006). A better knowledge of the disease symptoms can provide valuable information for insight into the disease spread and serve as a basis for disease management. 


\section{Materials and methods}

\section{Field surveys}

Cashew growing areas of Tanzania were surveyed during 2010 and 2011, at the flushing and flowering stages of the crop. The parameters, which were given particular emphasis, were the incidence and symptoms of the disease. The surveys were conducted in different ecological and cropping zones in collaboration with the staff of Naliendele Agricultural Research Institute, its substations at Nachingwea, Madangwa and Newala.

\section{Selection of locations}

The criteria used for the selection of the sites were those: i) representing different agroecological zones, ii) having typical cashew growing system in the area, iii) following the traditional as well as modern agricultural operations, and iv) growing exclusively field crops with particular emphasis on cashew. The following four zones were sampled: Zone 1, South half of Mtwara Rural and South-East of Newala - unimodal rainfall with annual precipitation exceeding $1000 \mathrm{~mm}$ and 6 months of growing season (November-April), low attitude, isohyperthemic temperatures, low fertility soils. Zone 2, North half of Mtwara Rural-unimodal rainfall averaging 600-1000 mm in 6 months (November-April), mid altitude, isohyperthemic temperatures, low fertility soils with medium moisture retaining capacity. Zone 3, Masasi, North and West parts of Newala-unimodal rainfall averaging 600-1000 $\mathrm{mm}$ in 6 months (December-April), low attitude, isohyperthemic temperatures, low fertility soils. Zone 4, South East of Mtwara Rural-bimodal rainfall pattern with annual precipitation of over $600 \mathrm{~mm}$, low altitude, growing season lasts 7 months (NovemberMay), high fertility alluvial soils.
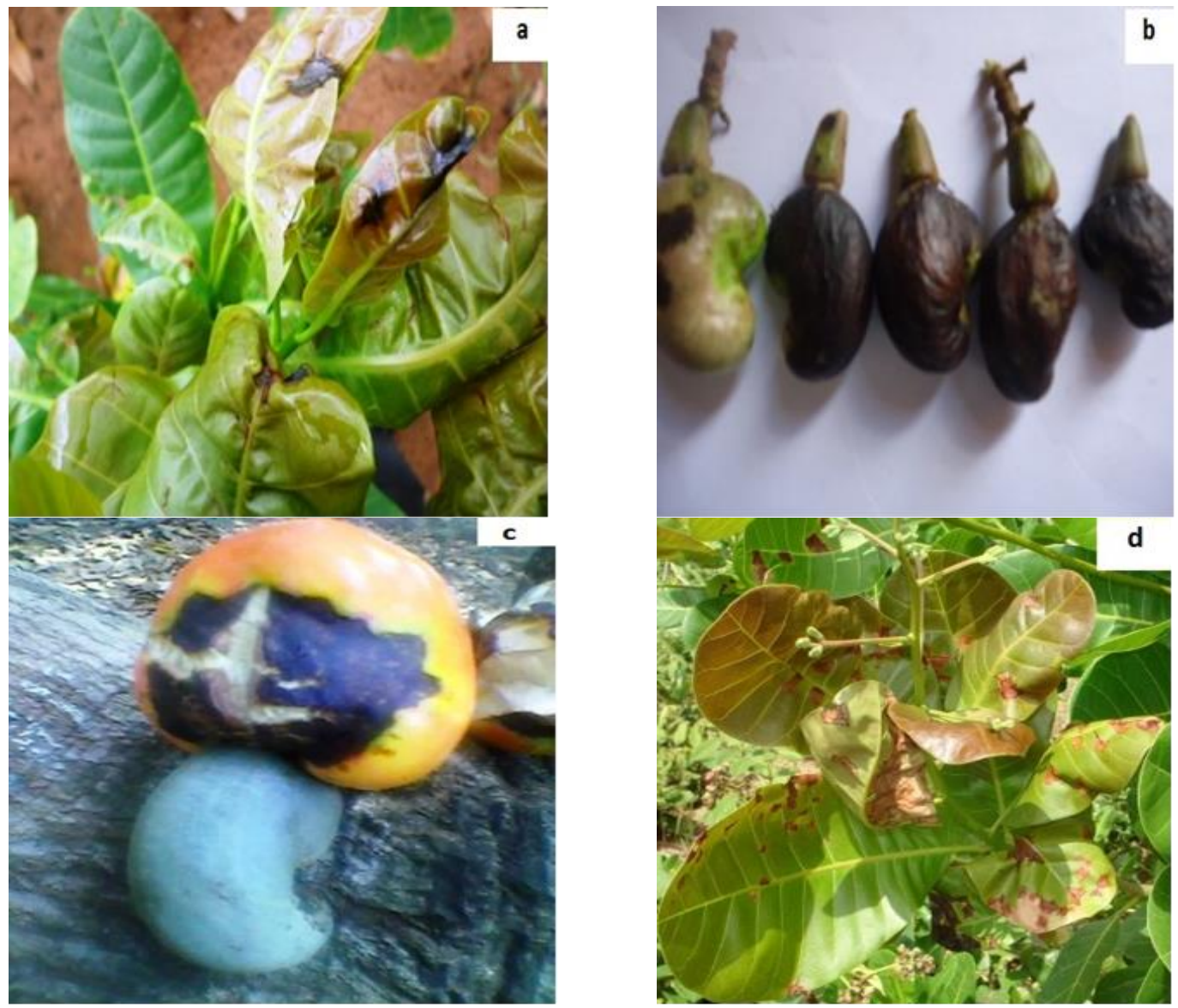

Fig 1 - Symptoms caused by Cryptosporiopsis sp. (cashew leaf and nut blight) a. Infected young tender cashew leaves with brown spots b. Infected cashew young nuts c. Infection on cashew pseudo-fruit. Panicle emerging from surrounding infected cashew shoots. 


\section{Sampling}

During sampling, factors such as host condition, growth stages, and disease development, were taken into consideration. Representative samples, based on visual symptoms of the disease were taken from each field at random as per methods described by Sukhatme (1954) and Yates (1960). Sampling for smaller units (less than 0.40 ha) was done either on a rectangular grid pattern or more frequently at regular intervals along the diagonals of the fields. For larger areas a group of 40 samples were drawn following an inverted $\mathrm{W}$ pattern. The sampling sites were approximately equidistant from each other along the sampling pathway. At each site, a specified number of plants at specified distance on the row were carefully examined and sampled. Plants of cashew were closely observed from the time of emergence till harvest and changes occurring in the diseased plants were noted carefully. Further, types of symptoms on leaves and nuts were also noted during the survey of different fields in Mtwara, Newala, Masasi and Nachingwea districts of Southern region. An infected part of plants i.e., leaves and nuts were collected from surveyed fields and packed in polythene bags. All samples were closely examined for the characteristic symptoms, mycelium, conidiophores and conidia using a compound microscope. Leaf pieces with fungus sporulatiom were transferred aseptically (Cappuccino \& Sherman et al. 2002) to potato dextrose agar (PDA) media and incubated at $25^{\circ} \mathrm{C}$ for 6 days. Leaf and nut blight fungus was purified by single spore isolation technique (Hubballi et al. 2010). The samples were forwarded to the Naliendele Agricultural Research Institute Plant Pathology Herbarium, Tanzania.

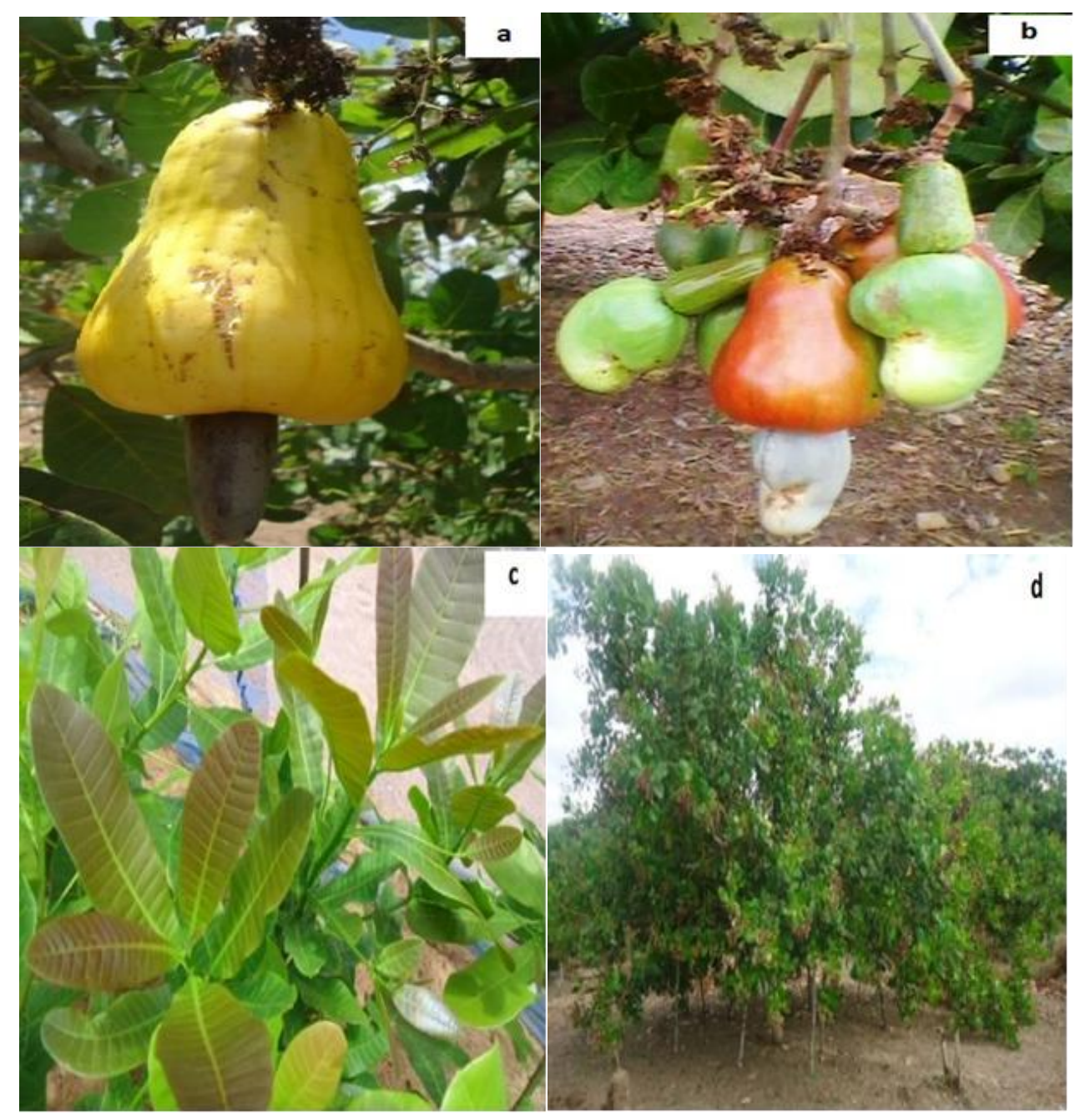

Fig 2 - Healthy leaves and nuts a. Healthy ripe cashew pseudo-fruit. b. Healthy nuts. c. Healthy cashew shoots. d. Cashew field. 


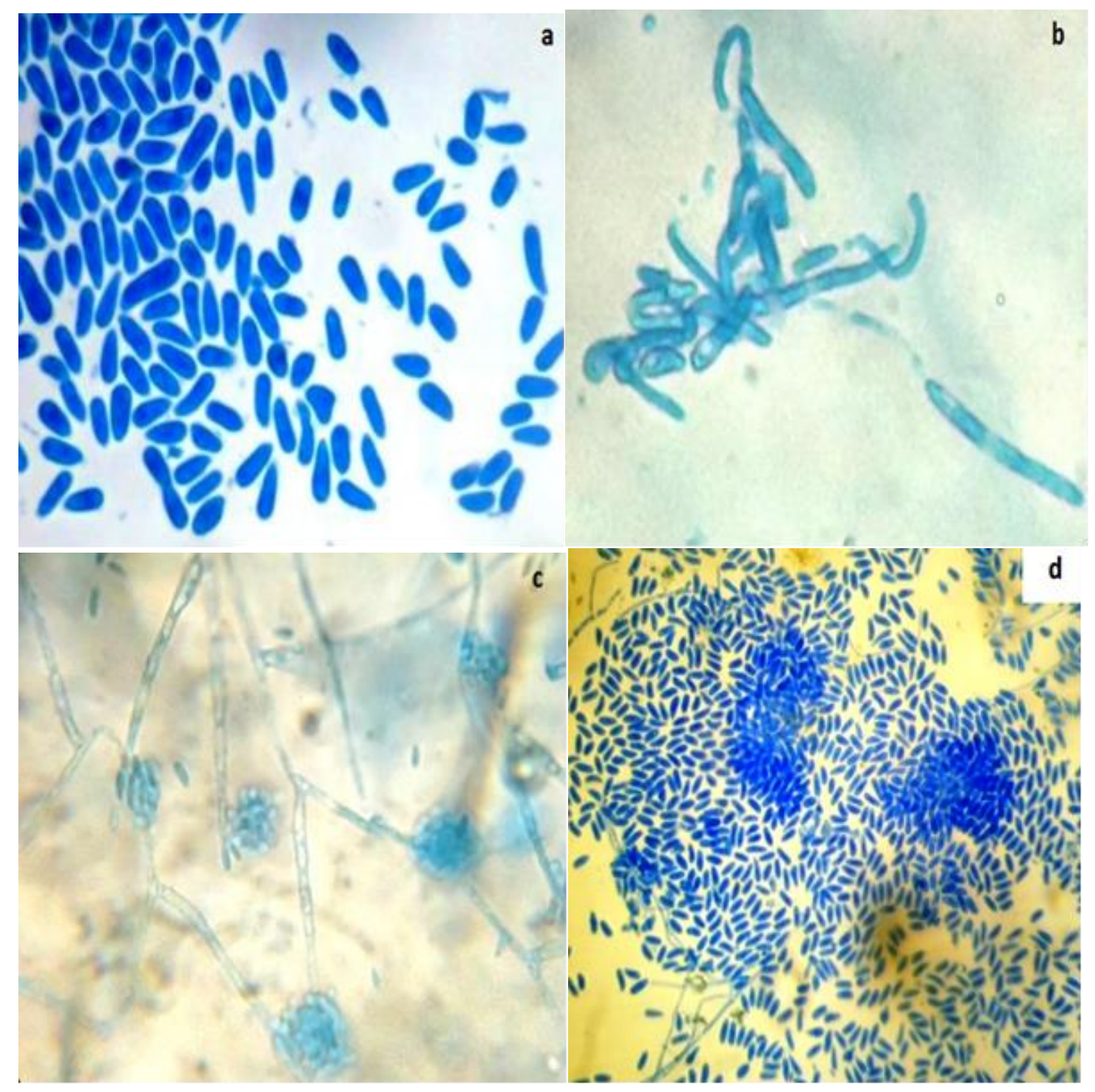

Fig 3 - Conidia and conidophores of Cryptosporiopsis sp. a. Conidia $(\times 400)$. b. Germinating conidia. c. Attachment of conidia on conidiophore. d. Conidia $(\times 100)$.

\section{Results}

Cryptosporiopsis sp. was found in all of the lesions. Infected plants became visible in all cashew trees irrespective of the age. First symptoms of the disease were chlorotic spots on both sides of the youngest tender leaves (Fig. 1a), although the abaxial surface had more infections than the adaxial side of young leaves. Leaf spots varied in size, shape and colour, within and between cashew varieties. The symptoms included depressed, brown, with a dark brown border, spreading necrotic lesions leading to a leaf blight symptom; circular, irregular 1-2 cm in diameter; irregular roughened or corky lesions with eruption and necrosis of epidermal tissue, sometimes localized along veins. Spots first appeared on upper half of the leaf near the tip (Fig. 1a). The diseased leaves curled and may fall off (Fig. 1a). Cashew leaf and nut blight infects young tender shoots, pseudofruits and nuts. Severely infected cashew trees did not produce panicles while infected nuts shrunk and were unmarketable. Infected pseudo-fruits burst open letting the juice out, which then attracted sucking insects and allowed other rots to develop, before eventually falling to the ground prematurely (Fig. 1c). Generally, the symptoms coincided with flushing of shoots and flowering in the months of July to December. Colonies of Cryptosporiopsis sp. were initially white becoming pale to dark brown in age on PDA. Conidiomata were numerous over the surface and were cream coloured and developed on foliar lesions on blighted shoots. Simple conidiophores have a swollen 
terminus bearing conidia. Conidia were smooth-walled and solitary. Conidia were slightly curved, aseptate, and arose from single phialides on vegetative hyphae or in conidiomata (Fig. 3c). They were brown to olive brown in colour, elliptical with tear-shaped ends. Conidia averaged $40.6 \pm 10.8$ $\mu \mathrm{m}$ in length and $10.7 \pm 2.3 \mu \mathrm{m}$ in width, with a length/width ratio of $4.0 \pm 1.4$ (Fig. 3a). Conidia of Cryptosporiopsis sp. germinated within 12 hours of landing on the leaf surface.

\section{Discussion}

The sampling revealed that cashew blight was prevalent in all the cashew growing areas. The samples were forwarded to the Naliendele Agricultural Research Institute Plant Pathology Herbarium, Tanzania where they were deposited as herbarium specimen Cryptosporiopsis sp. AA1AA10. Infected plants became visible in all cashew trees irrespective of the age. Sijaona et al. (2006) first reported damaging leaf and nut blight disease on young tissues of cashew in Tanzania. The symptoms included depressed, brown, with a dark-brown border, spreading necrotic lesions leading to a leaf blight symptom; circular, irregular 1-2 $\mathrm{cm}$ in diameter; irregular roughened or corky lesions with eruption and necrosis of epidermal tissue. Brown spots first appeared on upper half near the tip of the leaf. The diseased leaves curled and are sometimes defoliated. The abaxial surface had more infections than the adaxial part of young cashew leaves as observed. Generally, the symptoms coincided with flushing of shoots and flowering in the months of July to December.

\section{Acknowledgements}

We thank the German Federal Ministry for Economic Co-operation and Development (BMZ) for funding the cashew project, the International Centre of Insect Pathology and Ecology (ICIPE), Ministry of Agriculture and Food Security in Tanzania through Naliendele Agricultural Research Institute (NARI). We are grateful to CABI, UK, for providing a reference specimen.

\section{References}

Annual Cashew Research Report-ACRR 2006 - Chapter 3 Pathology Report pp. 98-99. Naliendele Agricultural Research Institute, Mtwara, Tanzania.

Cappuccino JG, Sherman N. 2002 - Microbiology. A Laboratory Manual. 6th Edition. Benjamin Cummings, CA.

Global Plant Clinic Issues - Volume 90, March 2010.

Hubballi, M., Nakkeeran, S. Raguchander, T. Anand T. and Samiyappan, R. 2010. Effect of environmental conditions on growth of Alternaria alternata causing leaf blight of noni. World Journal of Agricultural Sciences 6(2), 171-177.

Sijaona MER, Reeder RH, Waller JM. 2006 - Cashew leaf and nut blight - a new disease of cashew in Tanzania caused by Cryptosporiopsis spp. The Plant Pathology Journal 55, 576, Doi 10.1111/j.1365-3059.2006.01365.x.

Sukhatme PV. 1954 - Sampling theory of surveys with application. Iowa State University Press, pp. 491.

Yates F. 1960 - Sampling methods for census and surveys. 3rd Edition. Griffin, London, pp. 440. 